\title{
Decoding Angiotensin II Type 1 Receptor Allosteric Communication to Gq and $\beta$-arrestin
}

\section{Anita K. Nivedha, ${ }^{1, \ddagger}$ Yubo Cao, ${ }^{2, \ddagger}$ Sangbae Lee, ${ }^{1}$ Supriyo Bhattacharya, ${ }^{1}$ Stéphane A. Laporte ${ }^{2,3^{*}}$ and Nagarajan Vaidehi, ${ }^{1 *}$}

${ }^{1}$ Department of Computational \& Quantitative Medicine, Beckman Research Institute of the City of Hope, $1500 \mathrm{E}$. Duarte Road, Duarte, California 91010, USA

${ }^{2}$ Department of Pharmacology and Therapeutics, McGill University, Montreal, Quebec H3G 1Y6 Canada

${ }^{3}$ Division of Endocrinology and Metabolism, Department of Medicine, McGill University, Montreal, H4A 3J1

*To whom correspondence may be addressed. Email: NVaidehi@coh.org; stephane.laporte@mcgill.ca ${ }^{\ddagger}$ equal contribution

\section{ORCID:}

Anita K. Nivedha: 0000-0001-5272-7445

Yubo Cao: 0000-0002-9085-1153

Sangbae Lee: 0000-0003-3814-9604

Stéphane A. Laporte: 0000-0002-0633-543X

Nagarajan Vaidehi: 0000-0001-8100-8132

Classification: Biological sciences - Biophysics and Computational Biology

Keywords: G protein-coupled receptors (GPCRs), Molecular dynamics simulations, Angiotensin II type 1 receptor, AT1R, molecular signatures, microswitches, $G$ protein, $\beta$-arrestin2, ligand bias, allosteric communication, BRET.

Author Contributions: ${ }^{\ddagger}$ A.K.N. and Y.C. contributed equally to this work. N.V. and S.A.L. conceived the ideas and designed the experiments. A.K.N. and Y.C. executed the work and performed the analysis. S.L. performed MD simulations and some early analysis. S.B. contributed to analysis and discussions. N.V., A.K.N. wrote the paper with edits and suggestions from Y.C. and S.A.L. The authors declare no competing financial interest.

This PDF file includes:

Main Paper

Figures 1 to 4 


\begin{abstract}
The allosteric communication between the agonist binding site and the $\mathrm{G}$ protein or $\beta$-arrestin coupling sites in G protein-coupled receptors (GPCRs) play an important role in determining ligand efficacy towards these two signaling pathways and hence the ligand bias. Knowledge of the amino acid residue networks involved in the allosteric communication will aid understanding GPCR signaling and the design of biased ligands. Angiotensin II type I receptor (AT1R) is an ideal model GPCR to study the molecular basis of ligand bias as it has multiple $\beta$ arrestin2 and $\mathrm{Gq}$ protein biased agonists as well as three-dimensional structures. Using Molecular Dynamics simulations, dynamic allostery analysis, and functional BRET assays, we identified a network of residues involved in allosteric communication from the angiotensin II binding site to the putative $\mathrm{Gq}$ coupling sites and another network to the $\beta$-arrestin2 coupling sites, with 6 residues common to both pathways located in TM3, TM5 and TM6. Our findings unveil unique and common allosteric communication residue hubs for $\mathrm{Gq}$ and $\beta$-arr2 coupling by Angll ligands and suggests that some of these residues can be targeted to design biased AT1R ligands. Finally, we show through analysis of the inter-residue distance distributions of the activation microswitches involved in class A GPCR activation for ten different agonists, that these microswitches behave like rheostats with different relative strengths of activation, which we speculate could modulate the relative efficacy of these agonists toward the two signaling pathways.
\end{abstract}

\title{
Significance Statement
}

Knowledge of the residues involved in allosteric communication from the ligand binding site to the $G$ protein or $\beta$ arrestin ( $\beta$-arr) coupling sites in GPCRs will aid in understanding their role in mediating ligand bias. Using a combination of molecular dynamics simulations and functional signaling assays we have identified a network of residues involved in allosteric communication from the Angiotensin II (Ang II) binding site to the Gq and $\beta$-arr2 coupling sites in the Ang II type I receptor (AT1R). The residues in the allosteric network for $\beta$-arr2 coupling are distributed across multiple structural regions of AT1R compared to $\mathrm{Gq}$ coupling. The residues in the two networks show conserved chemical properties across class A GPCRs, demonstrating the importance of allosteric communication in modulating ligand bias.

\section{Main Text Introduction}

Angiotensin II (Angll) type 1 receptor (AT1R) belongs to the class A G protein-coupled receptor (GPCR) superfamily of membrane proteins that is activated by the octapeptide hormone Angiotensin II (Angll). Angll is part of the renin-angiotensin-aldosterone system responsible for controlling blood pressure and water retention via smooth muscle contraction and ion transport, and therefore forms an important drug target (1). Binding of Angll to AT1R leads to activation of heterotrimeric $\mathrm{G} \alpha q(\mathrm{Gq})$ family of $\mathrm{G}$ proteins, with subsequent engagement of $\beta$ arrestin2 ( $\beta$-arr2). The $\beta$-arr2 mediated signaling pathway may lead to pharmacologically desirable cardio protection, while over-activating the Gq coupled pathways leads to undesired cardiovascular effects (2). Therefore, agonists targeting AT1R that preferentially activate the $\beta$-arr2 signaling pathway over the Gq pathway commonly known as "biased agonists" are sought after for better therapeutic outcomes (3-9). Understanding the molecular mechanism of biased signaling and the amino acid residues involved in the information flow (or allosteric communication) from the agonist binding site to the $\mathrm{Gq}$ and $\beta$-arr2 coupling sites would aid the design of biased agonists.

Great strides have been made by recent structural studies on the structure and dynamics of AT1R with different peptide agonists (10-14). The structures of AT1R bound to a nanobody that stabilizes the active state of the receptor and with different biased agonists showed that the receptor adopts very similar conformations in the intracellular regions. However, there were differences observed in certain residue positions in the agonist binding site and in the sodium binding site that is known to be involved in activation of other class A GPCRs (14). An elegant study using double electron-electron resonance (DEER) spectroscopy demonstrated that biased agonists and Angll stabilize conformation ensembles that show distinct differences in the inter-residue distances between labeled residues in the intracellular region of AT1R (10). Single molecule force spectroscopy studies have shown differences in the off rates of the unbinding process of various biased agonists as well as in the number of conformational substates of AT1R when bound to $\mathrm{Gq}$ biased agonists compared to $\beta$-arr2 biased agonists (11). An all-atom Molecular Dynamics (MD) simulation study by Suomivuori et al. on AT1R bound to different biased agonists have shown that AT1R adopts two distinct conformations one of which is speculated to favor $\beta$-arr2 coupling and other Gq coupling (13). Using the residue rearrangements in the receptor, they designed and tested a biased agonist. Although these studies provide evidence for ligand specific effects in the intracellular $\mathrm{Gq}$ or $\beta$-arr2 coupling regions of AT1R, the mechanism of the allosteric communication from the agonist binding region to the $\mathrm{Gq}$ and $\beta$ arr2 coupling regions remains unclear. Identifying the residues involved in the allosteric communication would provide mechanistic insights into the ligand specific effects on $\mathrm{Gq}$ or $\beta$-arr2 coupling and thereby the ligand bias. 
Here, we used MD simulations for Angll bound AT1R in explicit lipid bilayer, combined with cellular functional BRET assays for measuring AT1R coupling to $\mathrm{Gq}$ and $\beta$-arr2, to quantitatively predict and test the residue networks that contribute to allosteric communication to $\mathrm{Gq}$ and $\beta$-arr2 coupling thus giving rise to signaling bias. Our results show that the allosteric communication in AT1R is mediated by a broad network of residues connecting the agonist binding site to the $\mathrm{Gq}$ or $\beta$-arr2 coupling sites. The network of residues involved in allosteric communication to $\beta$-arr2 is spread out across different structural regions (transmembrane helices TM5, TM6, TM7, helix8 and intracellular loop 1 or ICL1) of AT1R compared to the network communicating to the Gq coupling site that involves a majority of residues on TM5 and TM6. We identified six residues that are common to Gq and $\beta$-arr2 response $\left(\mathrm{I} 245^{6.40}, \mathrm{~S} 252^{6.40}, \mathrm{~F} 249^{6.44}, \mathrm{~F} 208^{5.5} \mathrm{H} 256^{6.51}\right.$ and R126 $\left.{ }^{3.50}\right)$. Despite being common, these residues had differential outcomes on each response when mutated to Ala. F208A $\mathrm{A}^{5.51}$ showed stronger coupling efficiency to $\mathrm{Gq}$ as compared to $\beta$-arr2, and as compared to wild type (WT). Although reduced coupling to both pathways compared to WT, F249A $\mathrm{A}^{6.44}$ also showed stronger coupling efficiency to $\mathrm{Gq}$ than $\beta$-arr2. On the other hand, R126A ${ }^{3.50}$ exhibited stronger coupling to $\beta$-arr2 than Gq compared to WT, while H256A $A^{6.51}$ also revealed better coupling efficiency to $\beta$ arr2 than to Gq, yet less efficiently in both reponses as compared to WT AT1R.

\section{Results}

Starting from the crystal structure of the partial agonist peptide S1I8 bound active state of AT1R (PDB: 6DO1) (12) we modeled ten different Angll-based peptide agonists, including Angll, SII, six $\beta$-arr2 biased, and two $\mathrm{G}$ protein biased agonists (shown in Table S1) into this structure. We used the crystal structure of S1I8-AT1R complex structure, because it was the only crystal structure of the active state of AT1R available at the beginning of this study. Follwing $50 \mathrm{~ns}$ of equilibration procedure for each system (described in the Methods section) we performed five runs each 400ns long of all-atom MD simulations in explicit lipid bilayer (details in Table S2). The resulting aggregated $2 \mu$ s of trajectories for each of the ten systems were used for further analysis.

\section{Residues involved in the allosteric communication from Angll binding site to the Gq protein or $\beta$-arr2 coupling sites}

We previously developed a computational method Allosteer to identify the residues involved in allosteric communication between stipulated sites in a GPCR (15). Allosteer involves calculation of the correlation (or mutual information) in torsion angle distribution for every pair of residues in a given GPCR. For each pair of residues with high mutual information we use graph theory method to calculate the shortest pathway with maximum mutual information. At the end of these calculations, every residue in the GPCR is assigned a "hub score" that quantitfies the number of allosteric communication pathways that pass through that residue (15-18). One could calculate the hub score for all the residues in the user-specified allosteric communication pathways from the agonist binding site to the $G$ protein or $\beta$-arrestin coupling sites. We showed that residues located in the allosteric communication pathways modulate the receptor activity depending on the ligand bound to the receptor $(15,17)$. Here, using Allosteer we predicted a set of 26 residues that are shown in Fig. 1A with high hub scores involved in allosteric communication between residues in the Angll binding site and those in the putative Gq protein coupling site. Similarly we predicted a set of 31 residues (Fig. 1C) with high hub scores to $\beta$-arr2 coupling site (see Methods for details).

The residues predicted to be involved in allosteric communication to the $\mathrm{Gq}$ or $\beta$-arr2 coupled sites were tested for their effect upon mutation to alanine (existing alanine residues were instead replaced with glycines) using BRET-based biosensors in cells (19-22). The AT1R activation of Gq pathway was measured using the downstream PKC BRET biosensor, while $\beta$-arr2 engagement was measured using an enhanced bystander BRET sensor, as previously described (19-23). The enhanced bystander BRET assay measures $\beta$-arr2 recruitment at the plasma membrane upon receptor activation, and will likely be sensitive to mutations regulating the receptor directly and/or conformationally driven interactions with $\beta$-arr2, including those affecting the phosphorylation of receptors (26). We first assessed how receptor expression affects the maximal response of each BRET sensor response by titrating DNA transfection of the wild-type AT1R in cells (see Supplementary text and Figs. S1A to S1D). Next, we expressed wild-type and mutant receptors with BRET biosensors to evaluate their coupling efficacies ( $E_{\max }$ (Fig. 1B and 1D), as well as the potency of Angll to promote responses in each pathway $\left(\mathrm{EC}_{50}\right)$ (Table S3). All mutant receptors expressed over $50 \%$ of WT (except for mutants $1245 \mathrm{~A}^{6.40}$ and T175A $\mathrm{A}^{\mathrm{ECL} 2}$ ) (Figs. 1B and 1D), implying that any loss of signaling responses could not be attributed to a defect in the mutant expression. We evaluated the effect of mutations on receptor activity by comparing their coupling efficacies in each pathway at saturating concentration of Angll where maximal receptor occupancy is reached. This minimizes potential confounding effects of mutations on Angll affinity, which would be mirrored in changes in $\mathrm{EC}_{50}$ as seen for some AT1R mutants (Table S3). 
Residues that showed a significant change in receptor activity (either positive or negative) for Gq coupling, when mutated, are shown in Figs. $1 \mathrm{~A}$ and $1 \mathrm{~B}$, while those which showed significant changes in $\beta$-arr2 recruitment are shown in Figs. 1C and 1D. We next assess quantitatively the performance of our prediction of allosteric hubs using the Receiver Operating Characteristic (ROC) Curve analysis shown in Figs. S3A and S3B. The area under the ROC curve is 0.7 for recovering the residues that affect receptor activity for $\mathrm{Gq}$ coupling and 0.6 for $\beta$ arr2 recruitment, which is a significant enrichment in comparison to random prediction and considering that predictions were made using sparse structural information, independent of experimental data. It is worth noting that that the Allosteer method is a system agnostic quantitative method and can be applied to identify allosteric communication networks in any protein without prior knowledge as we have illustrated in these publications $(18,24,25)$.

Allosteric Communication network to the Gq coupling site mainly passes through TM5 and TM6 while to $\beta$-arrestin2 spreads across different structural regions of AT1R

The majority of residues in AT1R involved in allosteric communication to the $\mathrm{Gq}$ coupling site are located in TM5 and TM6, followed by ICL2, TM3 and TM4 (Fig. 1A, Fig. S4), while residues that communicate to the $\beta$-arr2 coupling site are not only more in number, but also distributed more widely across TM5, TM6, TM7, h8 and ICL1 (Fig. 1C, Fig. S4). Analysis of the number of GPCR-G protein contacts in the three dimensional structure of $\mathrm{G}_{\mathrm{q} / 11}$ coupled muscarinic acetyl choline $\mathrm{M}_{1}$ receptor (26) shows a lower number of contacts than the GPCR- $\beta$-arrestin contacts in the structure of $\beta 1$-adrenergic receptor- $\beta$-arr1 complex (27) (Fig. S5A to S5C). The contacts with $\beta$-arr1 are distributed amongst TM5, TM6, ICL2, TM3, TM2, ICL1, TM7 and h8 region of the $\beta 1$-adrenergic receptor, while those with the $G_{q / 11}$ protein are found predominantly in ICL2, TM5, TM6 TM3 and h8 of $M_{1}$ muscarinic receptor. This could be a reason why the residues involved in the allosteric communication to $\beta$-arr2 coupling are also spread out across different structural regions of AT1R compared to the residues involved in allosteric communication to Gq.

Analysis of residues involved in allosteric communication network to $\mathrm{Gq}$ and $\beta$-arr2 in AT1R revealed that six residues located on TM3, TM5 and TM6 are common to both $\beta$-arr2 and Gq allosteric communication (Fig. 1). Because we obtained the necessary pharmacological parameters for the signaling effects of these residues when mutated to Ala for both of the two signaling pathways, this gave us the opportunity to determine to what extent their substitution biased the receptors' responses. When first looking at their coupling efficacies to $\mathrm{Gq}$ and $\beta$-arr2 $\left(\Delta \mathrm{E}_{\max }\right.$ Fig. S3C and S3E), we found that mutants F249A $A^{6.44}$ and F208A $A^{5.51}$ showed significantly more coupling to Gq as compared to $\beta$-arr2 in the mutants. F208 ${ }^{5.51}$ showed stronger coupling efficiency to $\mathrm{Gq}$ as compared to $\beta$-arr2, and as compared to WT. F249A ${ }^{6.44}$ also showed stronger coupling efficiency to Gq than $\beta$-arr2 but not compared to both the signaling pathways in the WT. However, H256 $\mathrm{A}^{6.51}$ and R126 $\mathrm{A}^{3.50}$ showed the contrary, and $1245 \mathrm{~A}^{6.40}$ and S252A ${ }^{6.47}$ revealed no significant differences between the two signaling pathways. When calculating signaling bias using differences in the relative activity as done previously (21), and despite potential caveats underscored earlier of using such an approach with mutant receptors, we found that while F249A ${ }^{6.44}$ and F208A ${ }^{5.51}$ became more

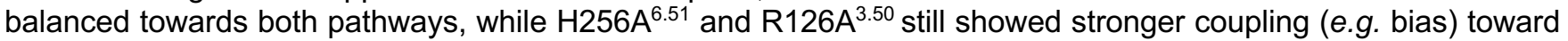
$\beta$-arr2 compared to $\mathrm{Gq}$ in the mutants (Fig. S3D and S3E). R126A ${ }^{3.50}$ exhibited stronger coupling to $\beta$-arr2 than $\mathrm{Gq}$ compared to WT, while H256 $A^{6.51}$ showed weaker responses in both pathways compared to WT AT1R. I245A $A^{6.40}$ and $S 252 A^{6.40}$ remained balanced. These findings suggest that despite these residues belonging to the common $\mathrm{Gq}$ and $\beta$-arr2 allosteric communication network, some nonetheless differentially contributed to each response, and can be targeted to generate functional selective AT1R. Obviously, other residues we identified at the unique allosteric $\mathrm{Gq}$ or $\beta$-arr2 hubs may also be targeted by mutagenesis to generate bias signaling AT1R mutants. However, their effects will need to be empirically determined in each complementary pathway to evaluate potential bias. Also, despite identifying key residues for the allosteric $\mathrm{Gq}$ and $\beta$-arr2 hubs, we cannot exclude the possibility that amino acids elsewhere in the receptor may also have differential bias signaling effects in AT1R when mutated to Ala or other residues. Indeed, in our prediction using Allosteer we calculate the hub score for each residue in the wild type AT1R, without considering their substitutions for other amino acids. Further work will be needed to establish the effects of such mutants and their putative biased nature.

\section{Residues involved in allosteric communication show conserved chemical characteristics across class A GPCRs}

To understand the broader functional relevance of the AT1R allosteric residue network to other class A GPCRs, we examined the conservation of their chemical characteristics (see Methods for definitions) among all class A GPCRs (Fig. S6) using the GPCR-SAS webserver (28). 16 out of 26 residues that showed significant change in AT1R coupling to $\mathrm{Gq}$ are in the top conserved group of amino acids amongst all class A GPCRs, with 11 of those strictly conserved. Of the remaining 10 allosteric hub residues to $\mathrm{Gq}$ coupling, 5 belong to the second most conserved group of amino acids (Fig. S6A). Similarly, 17 out of 31 residues that showed significant change 
bioRxiv preprint doi: https://doi.org/10.1101/2021.05.17.444454; this version posted May 17, 2021. The copyright holder for this preprint (which was not certified by peer review) is the author/funder. All rights reserved. No reuse allowed without permission.

in AT1R coupling to $\beta$-arr2 belong to the most conserved group of amino acids in this position, with 8 being strictly conserved. Of the remaining 14 hubs, 8 belong to the second most conserved group of amino acids at this position (Fig. S6B). The evolutionary conservation of the residues involved in allosteric communication from the agonist binding site to the $G$ protein and $\beta$-arr2 coupling sites and across all class A GPCRs further underscores the importance of these amino acids for the function of class A GPCRs.

A

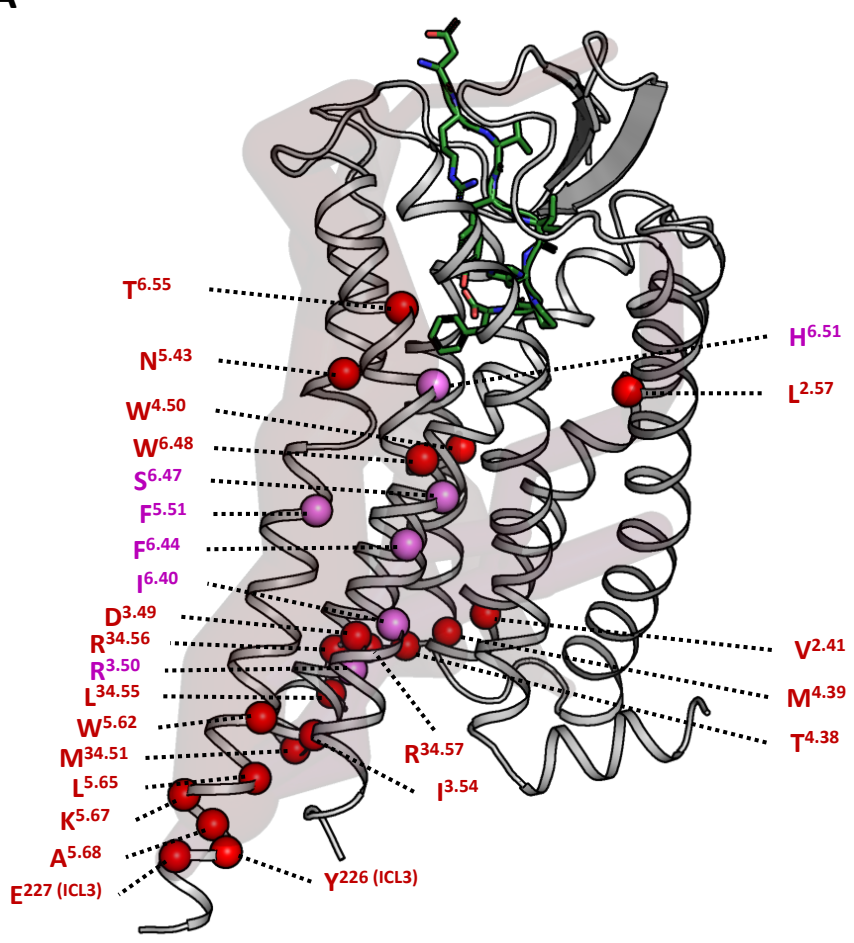

C

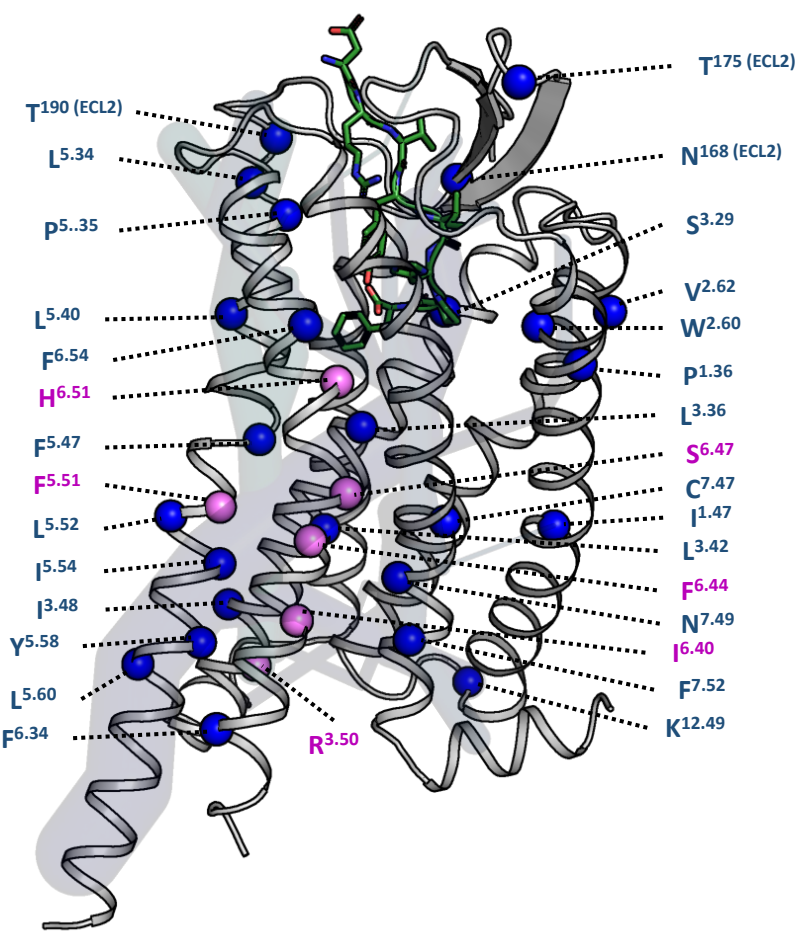

B

\begin{tabular}{|c|c|c|c|c|c|}
\hline BW \# & Mutant & Gaq Activation (\%WT) & S.D. & Significance & Expression (\%WT) \\
\hline \multicolumn{2}{|c|}{ Wildtype } & 100.0 & 0.00 & N/A & 100.0 \\
\hline 4.38 & T141A & 38.6 & 2.38 & $* * * *$ & 126.9 \\
\hline ICL2 & R140A & 55.3 & 2.34 & $* * * *$ & 122.1 \\
\hline ICL2 & M134A & 56.1 & 1.84 & $* * * *$ & 122.9 \\
\hline 3.49 & D125A & 63.4 & 2.71 & $* * * *$ & 80.9 \\
\hline 3.54 & I130A & 70.5 & 2.37 & $* * * *$ & 134.8 \\
\hline 6.51 & H256A & 71.4 & 3.19 & $* * * *$ & 93.8 \\
\hline 3.50 & R126A & 71.5 & 4.02 & $* * * *$ & 100.4 \\
\hline 5.65 & L222A & 71.8 & 2.15 & $* * * *$ & 53.5 \\
\hline 4.39 & M142A & 76.8 & 3.34 & $* * * *$ & 67.4 \\
\hline 2.57 & L81A & 81.1 & 1.71 & $* * * *$ & 90.3 \\
\hline 6.48 & W253A & 83.2 & 2.68 & $* * * *$ & 81.6 \\
\hline 6.44 & F249A & 84.9 & 2.18 & $* * * *$ & 88.0 \\
\hline ICL3 & E227A & 85.9 & 3.39 & $* * * *$ & 66.2 \\
\hline ICL2 & L138A & 86.4 & 2.79 & $* * * *$ & 105.3 \\
\hline 5.43 & N200A & 87.9 & 2.05 & $* * *$ & 93.5 \\
\hline 4.50 & W153A & 87.9 & 3.92 & $* * *$ & 100.2 \\
\hline ICL2 & R139A & 89.2 & 4.04 & $* * *$ & 103.3 \\
\hline 2.41 & V65A & 89.5 & 4.96 & $* *$ & 105.0 \\
\hline 5.62 & W219A & 104.9 & 3.26 & ns & 110.6 \\
\hline ICL3 & Y226A & 105.2 & 2.79 & ns & 126.2 \\
\hline 6.55 & T260A & 105.9 & 3.67 & ns & 77.5 \\
\hline 5.51 & F208A & 106.9 & 2.98 & ns & 117.1 \\
\hline 5.67 & K224A & 107.4 & 3.06 & ns & 135.5 \\
\hline 6.40 & I245A & 107.8 & 3.36 & $*$ & 44.5 \\
\hline 5.68 & A225G & 108.9 & 2.66 & $*$ & 116.1 \\
\hline 6.47 & S252A & 110.9 & 3.00 & $* * *$ & 109.6 \\
\hline & & & & & \\
\hline
\end{tabular}

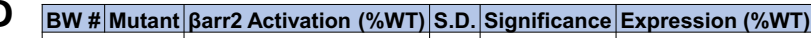

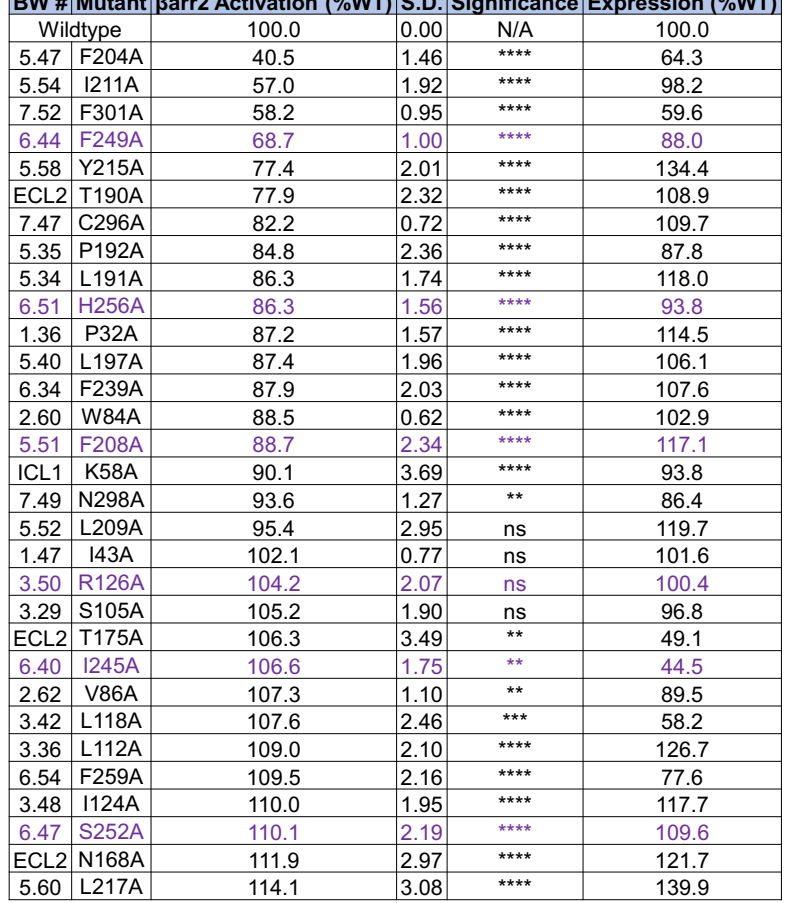


Figure 1. (A) Angll (green stick representation) bound AT1R with residues predicted to be involved in allosteric communication to the Gq protein coupling interface (red and purple spheres) that also showed a significant change experimentally in $\mathrm{Gq}$ protein coupling upon mutation to alanine using BRET assays. Hub residues are indicated using BW numbering. Residues in purple are hubs common to both $\mathrm{Gq}$ protein and ßarr2 signaling. The major allosteric communication pipelines housing the hubs contributing to $\mathrm{Gq}$ protein signaling are shown in shades of transparent red pipe representation. (B) List of allosteric hubs for $\mathrm{Gq}$ signaling and BRET measurements on wild type and AT1R mutants expressed with the PKC-c1b sensor in HEK293SL cells and stimulated with various concentrations of Angll to generate concentration-response curves to calculate maximal $\mathrm{Gq}$ coupling and effector activation (Emax). Data represent mean \pm s.d. from 3 independent experiments, and one-way ANOVA followed by Dunnett's multiple comparisons test were performed where: ${ }^{* * *}=p<0.0001,{ }^{* * *}=p<0.001,{ }^{* *}=p<0.01,{ }^{*}=p<0.05$, $\mathrm{n}=3$. Expression level of receptors determined through whole-cell ELISA is also reported. (C) Angll (green stick representation) bound AT1R with residues predicted to be involved in allosteric communication to the $\beta$-arr2 coupling interface (blue and purple spheres) that also showed a significant change in $\beta$-arr2 coupling upon mutation using BRET assays. The major allosteric communication pipelines to ßarr2 interface are shown in shades of transparent blue pipe representation. (D) List of allosteric hubs for ßarr2 activation and BRET measurements on wild type and AT1R mutants expressed with $\beta$-arr2-Rlucll and rGFP-CAAX in HEK293SL cells and stimulated with various concentrations of Angll generate concentration-response cuvres to calculate maximal $\beta$-arr2 recruitment to receptors (Emax). Data represent mean \pm s.d. from 3 independent experiments, and one-way ANOVA followed by Dunnett's multiple comparisons test were performed where: ${ }^{* * * *}=p<0.0001,{ }^{* * *}=p<0.001,{ }^{* *}=p<0.01,{ }^{*}=p<0.05$, $\mathrm{n}=3$. Expression level of receptors determined through whole-cell ELISA is also reported.

\section{Relative allosteric communication pipeline strengths account for differences in ligand bias}

Recently (17) we advanced the Allosteer method to compute molecular level ligand bias, termed "molecular bias". We defined the molecular bias as the ratio of strengths of allosteric communication to the Gq protein interface and the $\beta$-arr2 interface for a test ligand compared to that of a reference ligand (see Methods). Our prior studies using this method for various biased and reference agonists to $\beta_{2}$-adrenoceptor, $k$-opioid receptor and serotonin receptors demonstrated good correlation with experimentally calculated ligand bias factors (17). We applied the same method to estimate the ligand bias of several agonists to AT1R, using Angll as the reference agonist. The experimental bias factors measured using Angll as the reference agonist were taken from literature $(29,30)$. As seen in Fig. 2, the calculated molecular ligand bias correlates qualitatively well with the experimental bias factors for both $\mathrm{Gq}$ protein biased and $\beta$-arr2 biased agonists. This qualitative correlation provides evidence that an atomistic property such as allosteric communication strength is one of the contributing factors that potentiates the ligand bias observed in agonist-receptor pairings. The details of the method used to calculate the ligand bias based on Allosteer and caveats of the method are discussed in the Supporting Information.

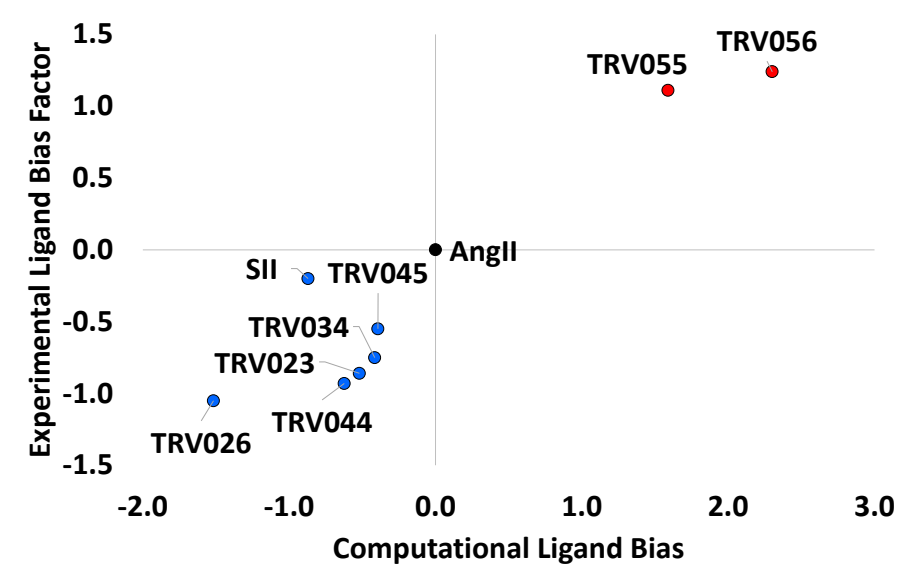

Figure 2. Calculated molecular ligand bias compared to experimental ligand bias factor of $\mathrm{Gq}$ or $\beta$-arr2 biased agonists for AT1R. The red dots represent $\mathrm{Gq}$ biased agonists and the blue dots represent $\beta$-arr2 biased agonists. Experimental bias factor values were taken from (29).

Spatiotemporal heatmap of agonist-AT1R contacts show differences in the binding site features of $\beta$-arr2 biased agonists versus $\mathrm{G} q$ biased agonists 
Here our goal was to analyze the agonist-specific residue contacts in the binding site of the ten agonist peptides studied here. All the ten agonists are peptide derivatives of the octapeptide Angll and the amino acid positions in the peptides are numbered 0 to 8 (Fig. S7A). The spatiotemporal heatmap in Fig. S7B shows the complete list of agonist-AT1R residue contacts and their persistence calculated from the MD simulation trajectories. The persistence of a ligand-protein residue contact is the percentage of MD snapshots that contain the agonistreceptor contact. The agonist-receptor contacts that show a significant difference in persistence among $\beta$-arr2 biased agonists compared to $\mathrm{Gq}$ biased agonists or Angll are likely to contribute to the functional selectivity of agonists. For each receptor-agonist contact we calculated the average persistence across the six $\beta$-arr2 biased agonists and the same across the two $\mathrm{Gq}$ biased agonists. We identified the agonist-AT1R contacts showing a difference in average persistence greater than $10 \%$ (which is persistence over a time period of 200ns) as functionally selective to $\beta$-arr2 or Gq. As shown in Fig. 3A, the agonist-AT1R contacts that are postulated to contribute to functional selectivity of $\beta$-arr2 agonists are mostly located in the $\mathrm{N}$-terminus and TM7, followed by ECL2, TM6 and TM4 with a small percentage of contacts in TM1. On the other hand, the agonist-AT1R contacts that we attribute to functional selectivity of the Gq biased agonists are predominantly located in TM3 and ECL2, followed by TM6, the N-terminus and TM5. 5\% of contacts is located in ECL3 and TM7. Residues in TM7 show a difference between $\beta$-arr2 biased agonists and $\mathrm{Gq}$ biased agonists. Our results could, however, be skewed towards $\beta$-arr2 agonists since we have six $\beta$-arr2 biased agonists compared to only two $\mathrm{Gq}$ biased agonists. Figs. 3B and $3 \mathrm{C}$ show the agonist-AT1R residue contacts that show higher persistence in TRV026 or in TRV056 compared to Angll. A large percentage of functional selective contacts for TRV026 compared to Angll are located in TM7 while they come from ECL2, TM5 and TM6 for the Gq biased TRV056. Although these agonist-AT1R contacts are speculated to contribute to functional selectivity, they have not been shown to be causative. 
A Distribution of binding site residues showing increased persistence with ßarr2-biased agonists

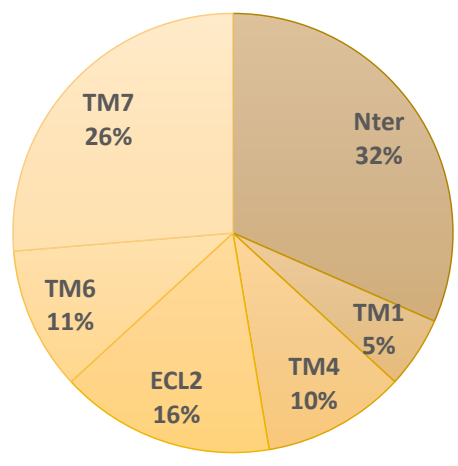

B

Functional selective residue contacts for TRV026

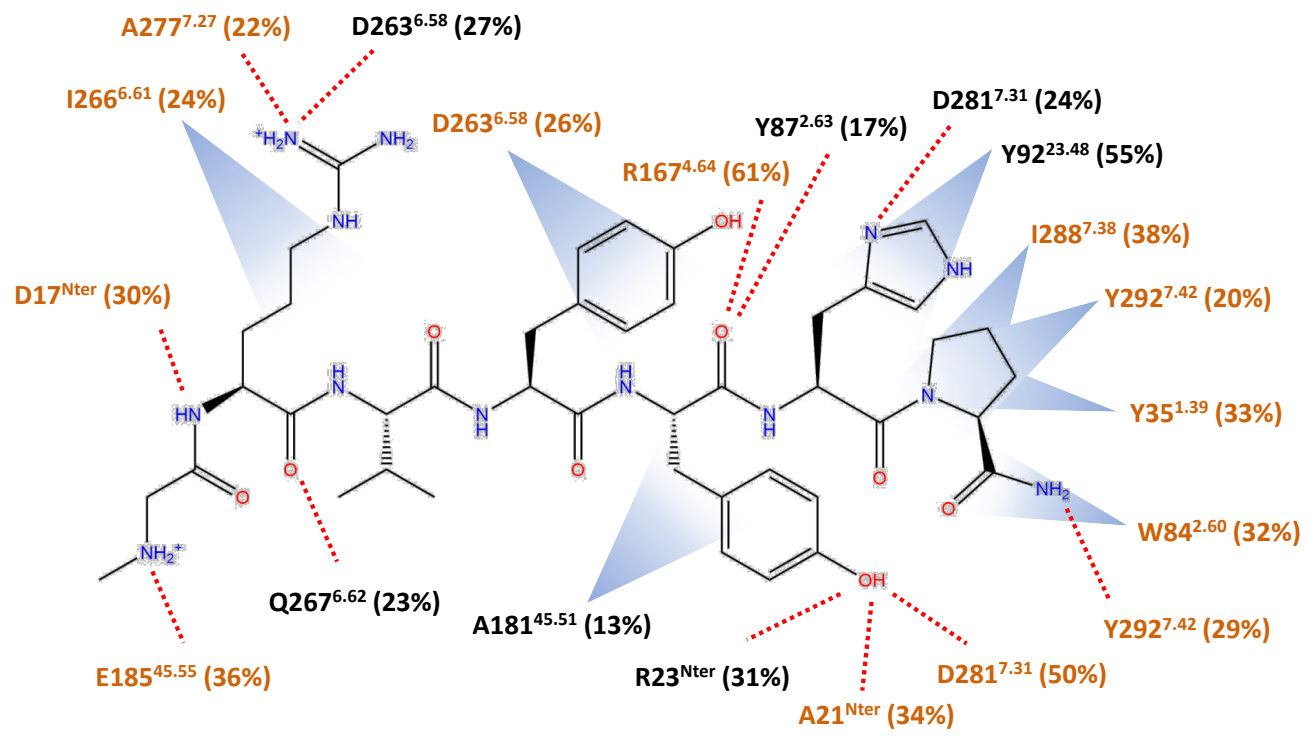

C

Functional selective residue contacts for TRV056

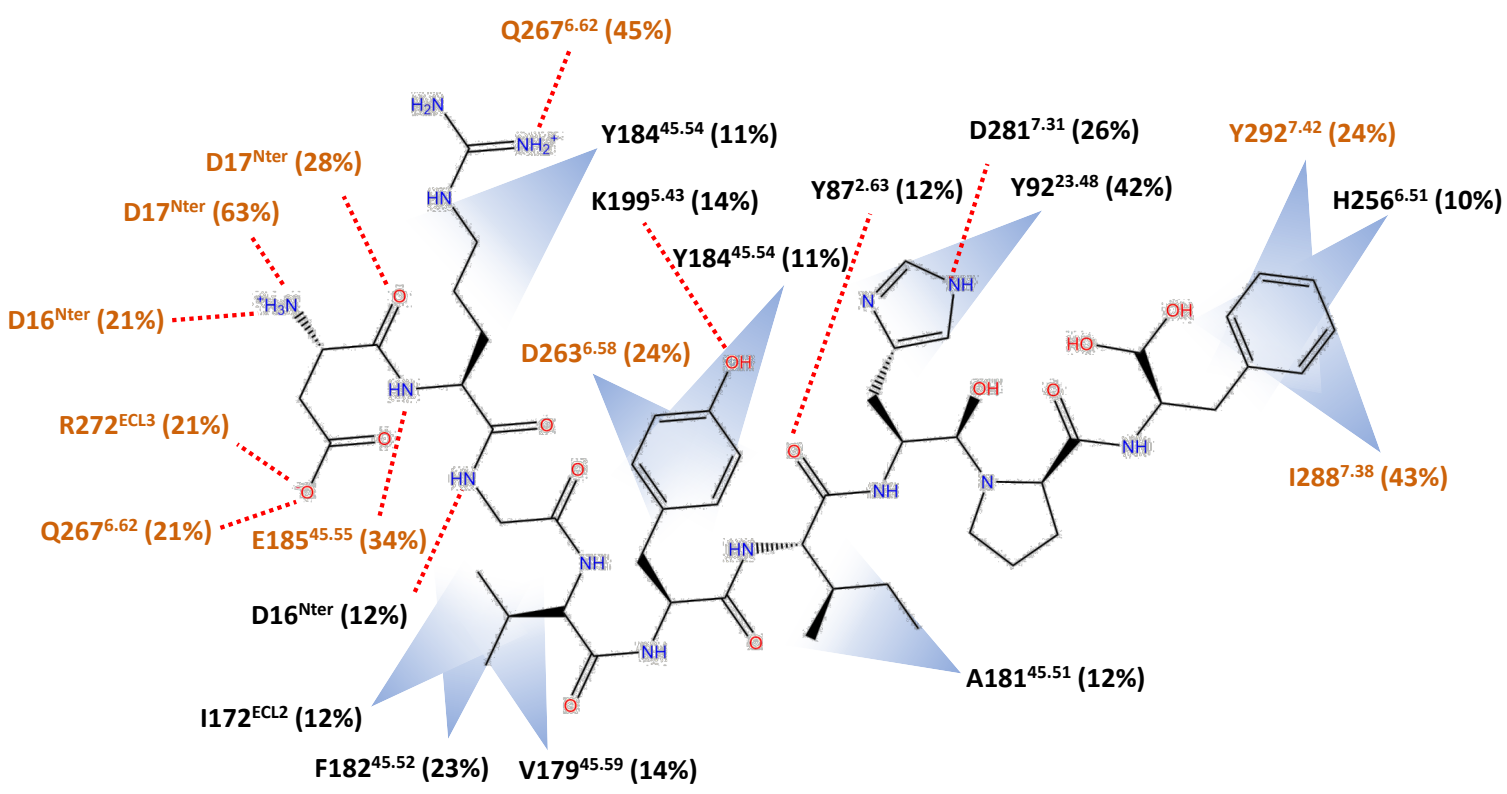


bioRxiv preprint doi: https://doi.org/10.1101/2021.05.17.444454; this version posted May 17, 2021. The copyright holder for this preprint (which was not certified by peer review) is the author/funder. All rights reserved. No reuse allowed without permission.

Figure 3. (A) Piecharts showing the percentage of agonist-AT1R contacts in each structural region of AT1R. Left: these receptor-agonist contacts show increased (greater than $10 \%$ difference which is equivalent to $200 \mathrm{~ns}$ in persistence) persistence amongst six $\beta$-arr2 biased agonists compared to the two Gq-protein biased agonists. Right: these receptor-agonist contacts show increased persistence in the two $\mathrm{Gq}$ protein biased agonists compared to the six $\beta$-arr2 biased agonists. (B) TRV026-AT1R residue contacts that show functional selectivity to TRV026. These residue contacts show increased persistence with TRV026 compared to Angll in AT1R and the difference in persistence is shown in parenthesis. Residue contacts observed exclusively in TRV026-AT1R dynamics and not present in Angll are shown in brown text. Polar contacts are indicated using red dashed lines and Van der Waals contacts are shown in blue shaded triangles. (C) TRV056-AT1R residue contacts with increased persistence for Gq biased TRV056 compared to Angll in AT1R. Residue contacts observed only in TRV056-AT1R dynamics and not present in Angll-AT1R dynamics are shown in brown text.

Activation microswitches behave as rheostats and show differential levels of activation in the presence of different agonists

Comparative analysis of inter-residue distances between inactive and active state structures of class $A$ GPCRs showed significant changes in certain inter-residue distances known as "activation microswitches" $(31,32)$. Contraction of the inter-residue distances between $N / S^{3.35}, D^{2.50}$ and $N^{7.46}$ collectively known as the sodium binding site, distance between $P^{5.50}$ and $F^{6.44}$ in the TM5-TM6 interface and the distance $Y^{5.58}-Y^{7.53}$ are three wellcharacterized "activation microswitches" in class A GPCRs (Fig. 4A). Some of the class A GPCRs show changes in activity towards $G$ protein coupling in response to sodium ion concentration (33). Crystal structures of some class A GPCRs showed the presence of sodium ions in the inactive state structures typically nested between residues $\mathrm{S}^{3.35}, \mathrm{~N}^{3.39}, \mathrm{D}^{2.50}$ and $\mathrm{N}^{7.46}$. The sodium ion binding site characterized by these residues shrinks upon receptor activation. However, Wingler et al. (14) showed that AT1R has no sensitivity in its activity to sodium ion concentration. Their crystal structure of Angll-AT1R active state complex showed outward movement of N111 3.35 compared to TRV026 or TRV023 bound AT1R active state structures. This resulted in an expansion of the putative sodium binding site in Angll-AT1R compared to antagonist bound inactive state structure of AT1R or even the TRV026-AT1R active state structure.

Activation microswitches are thought of as "on or off" binary state switches, a concept emerging from the analysis of static structures. Taking the ensemble approach of GPCR conformations, we posited that the activation microswitches for a ligand-receptor pairing could exhibit a rheostat like behavior with different levels of activation in each microswitch (34). To examine the effect of biased ligands on activation microswitches we calculated the inter-residue distance distribution from the $2 \mu \mathrm{s} \mathrm{MD} \mathrm{simulation} \mathrm{trajectories} \mathrm{for} \mathrm{each} \mathrm{of} \mathrm{the} \mathrm{ten} \mathrm{agonists} \mathrm{(Figs.} \mathrm{4B} \mathrm{and}$ S8). For each microswitch and for each agonist, we also calculated the percentage of MD snapshots (frequency) that move away from distances typified by the endogenous agonist Angll bound active state crystal structure. These frequencies show the percentage of snapshots that are neither in the fully active state or the inactive state (Fig. 4C). All the MD simulations show increased flexibility in the receptor when bound only to an agonist and in the absence of a G protein or nanobody. As a result, in the heatmap shown in Fig. 4C we observed that different agonists show a combination of different levels of occupancy of conformations that are intermediate to active and inactive states of each microswitch despite initiating the MD simulations from the same starting conformation. Our observations imply that the activation microswitches act as rheostats with different levels of activation rather than as binary on or off switches.

Angll-AT1R complex shows the most flexibility in the distance distributions of all microswitches compared to $\beta$-arr2 or $\mathrm{Gq}$ biased agonists as seen in Fig. 4B and Fig. S8. For example, Angll shows a small peak beyond $11.7 \AA$ (distance in the inactive state structure PDB ID: 4YAY) in the $Y^{5.58}-Y^{7.53}$ microswitch distance as shown in Fig. 4B. TRV055-AT1R complex also shows similar level of flexibility as Angll in the microswitches (Fig. S8). The $\beta$-arr2 biased agonists TRV026, TRV027 and TRV044 show a peak at the contracted $\mathrm{D}^{2.50}-\mathrm{N}^{7.46}$ distance around $3.3 \AA$ similar to the distance observed in the active state crystal structures of TRV026-AT1R (3.3A) and TRV023AT1R $(3.1 \AA)$ as opposed to $4.1 \AA$ observed in the Angll-AT1R active state structure. There is little variation in the frequency of the $D^{2.50}-\mathrm{N}^{3.35}$ microswitch across the $\beta$-arr2 and $\mathrm{Gq}$ protein biased agonists. This could be because for the $\beta$-arr2 biased agonists there is little change in the $D^{2.50}-\mathrm{N}^{3.35}$ distance observed in the active state crystal structure of TRV026-AT1R complex compared to the inactive state crystal structure of AT1R. In the Angll-AT1R complex there is a minor peak in the $\mathrm{D}^{2.50}-\mathrm{N}^{3.35}$ distance distribution that comes from a small conformation ensemble showing outward movement of residue $\mathrm{N} 111^{3.35}$ in our MD simulations (MD simulations were started from the active state conformation extracted from S118:AT1R structure which has the N111 3.35 facing inside the TM bundle). This leads to the orange cell in the heatmap for Angll (Fig. 4C). In summary, with just the agonist bound, the activation microswitches do not show a definitive pattern of the level of activation of each microswitch among the $\beta$-arr2 biased agonists (Fig. 4C). Instead, they show ligand specific combination of the level of activation of microswitches. In addition, the extent of activation of each microswitch is also ligand specific with rheostat-like behavior. This is 
understandable since the efficacy of these agonists towards $\beta$-arr2 coupling arises from an ensemble of conformations with different levels of activation of each of the microswitches.

A

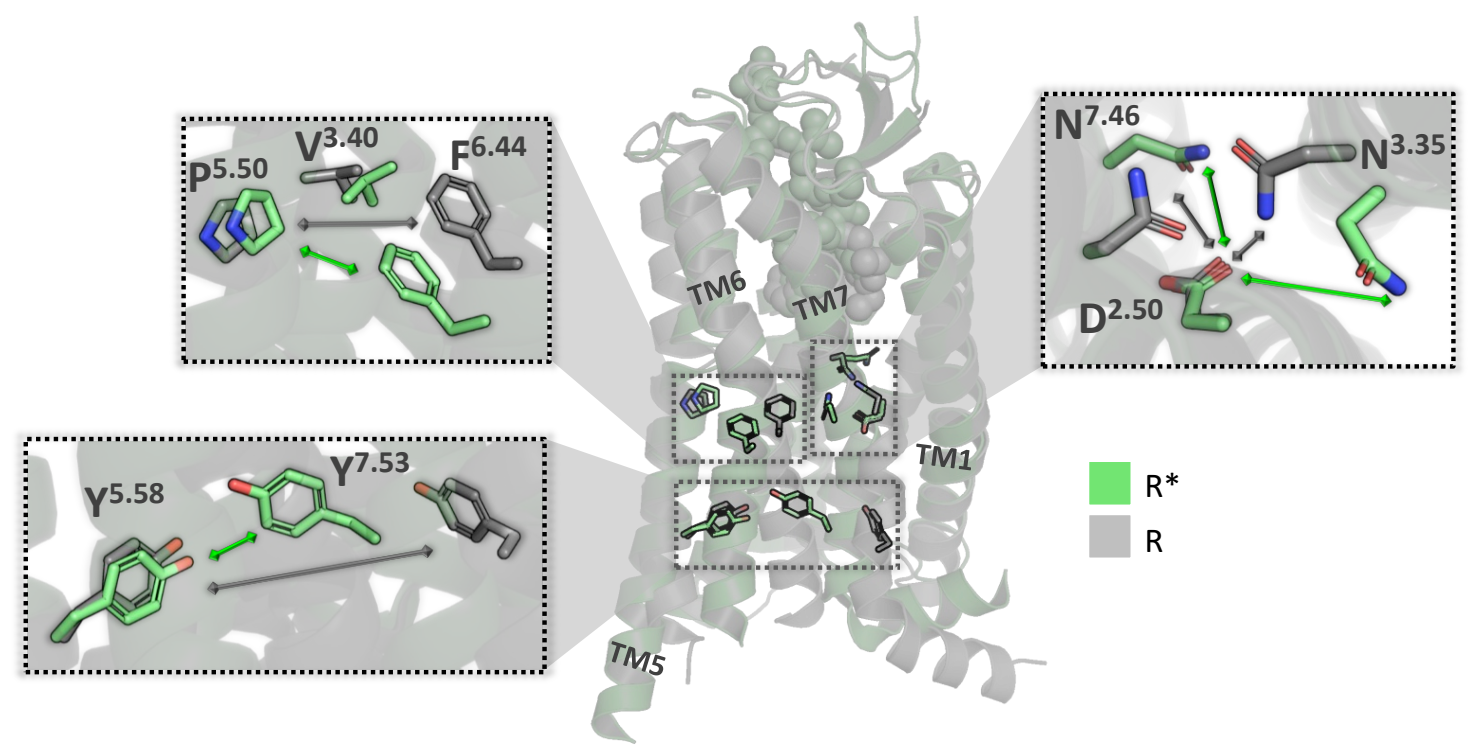

B
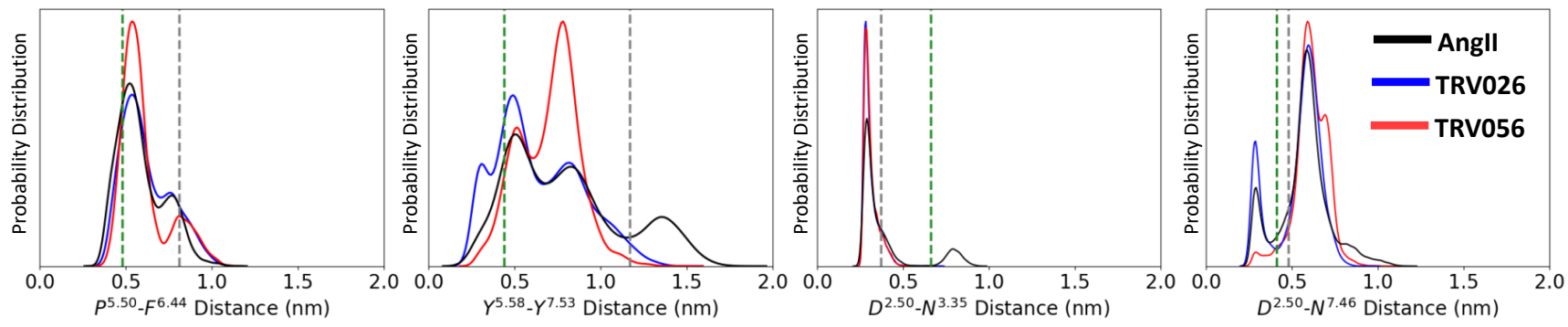

C

\begin{tabular}{|c|c|c|c|c|c|c|c|c|c|c|}
\hline $\begin{array}{l}\text { Activation } \\
\text { Microswitch }\end{array}$ & 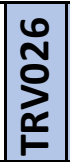 & 胥 & 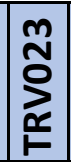 & 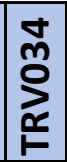 & 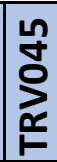 & No & $\underset{\mathbf{S}}{\mathbf{J}}$ & $\overline{\bar{n}}$ & 资 & 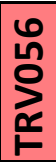 \\
\hline$P^{5.50}-F^{6.44}$ & & & & & & & & & & \\
\hline$Y^{5.58}-Y^{7.53}$ & & & & & & & & & & \\
\hline$D^{2.50}-N^{7.46}$ & & & & & & & & & & \\
\hline$D^{2.50}-N^{3.35}$ & & & & & & & & & & \\
\hline
\end{tabular}

\section{Frequency of active-intermediate conformations}

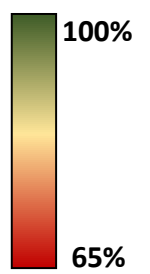

Figure 4. Class A GPCR activation microswitches show distinct patterns of activation with different biased agonists: (A) Four inter-residue distances that characterize GPCR activation known as activation microswitches are shown in the central figure. The green cartoon is the nanobody bound active state structure of Angll bound AT1R, $R^{*}$ (PDB: 6OS0) and the grey structure is the antagonist bound inactive state of AT1R, R (PDB: 4YAY). (B) The population density distribution of the inter-residue distances in the microswitches during the MD simulations of AT1R with various biased and balanced agonists are shown. The distances measured in AT1R are P207.50 $\mathrm{F} 249^{6.44}, \mathrm{Y} 215^{5.58}-\mathrm{Y} 302^{7.53}, \mathrm{D} 74^{2.50}-\mathrm{N} 295^{7.46}$ and $\mathrm{D} 74^{2.50}-\mathrm{N} 111^{3.35}$. The green and grey dashed lines show the corresponding inter-residue distances in the active (PDB: 6OS0) and inactive (PDB: 4YAY) state crystal structures respectively. (C) Heatmap of microswitch distances showing the level of activation for the different agonists when no nanobody or $\mathrm{G}$ protein is bound to the receptor, obtained by comparing to reference values obtained from the fully active state AT1R crystal structure (PDB: 6OS0).

\section{Discussion}


Allosteric communication from the extracellular agonist binding site to the intracellular core of GPCRs is one of the key molecular factors contributing to ligand efficacy towards $G$ protein and $\beta$-arrestin signaling pathways. AT1R is an ideal model GPCR system to study the molecular basis of allosteric communication bias, because it has available, $\mathrm{Gq}$ protein and $\beta$-arr2 biased agonists with experimentally measured bias factors, and crystal structures of nanobody-bound AT1R active state.

Starting from the nanobody bound AT1R crystal structures, we performed MD simulations in the presence of ten different agonists to extract the resulting structural differences in the receptor. Using a combination of the computational method Allosteer on the MD trajectories and BRET functional assays, we have enumerated a network of AT1R residues involved in the allosteric communication from the extracellular Angll binding site to the $\mathrm{Gq}$ coupling site and another network to the $\beta$-arr2 coupling site. Majority of these residue positions are either conserved or conservative replacements among class A GPCRs showing the functional importance of these positions. While the allosteric residue network to the Gq coupling site are largely localized on TM5 and TM6, those that communicate to the $\beta$-arr2 coupling site pass through TM5, TM6, TM7, h8 and ICL1, indicating a wider residue network covering more structural regions of the receptor than for $\mathrm{Gq}$ coupling. We speculate that this could be one of the reasons why it is more difficult to tune down $\beta$-arr2 mediated signaling than Gq signaling in AT1R (30). Our study showed that mutation of residues F249A ${ }^{6.44}$ and F208 $A^{5.51}$ increased coupling efficiency to $\mathrm{Gq}$ compared to

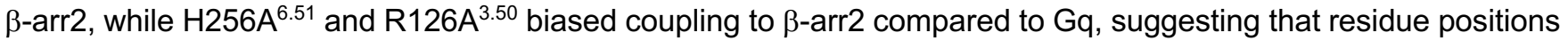
we identified in the common and specific $\mathrm{Gq}$ protein and $\beta$-arr2 hubs could be targeted to engineer AT1R biased mutants using alanine and/or non-alanine amino acid substitutions. We defined the molecular level ligand bias as the ratio of strength of allosteric communication to the Gq protein interface and the $\beta$-arr2 interface for a test ligand compared to a reference ligand. The molecular bias thus calculated shows a qualitative correlation with experimental bias factors demonstrating that allosteric communication is one of the factors influencing ligand bias. One caveat of our approach is that we translated the $G$ protein and $\beta$-arrestin binding site residues from single snapshot structures of other class A GPCRs. Also, our prediction of the residue network using Allosteer is based on the MD simulations of the wild type AT1R, without considering their substitutions for other amino acids. This is perhaps why, for instance, we did not capture AT1R mutants such as $D 74 \mathrm{~N}^{2.50}$ and $N 111 \mathrm{G}^{3.35}$ which have previously been shown to be biased $(35,36)$.

The spatiotemporal heatmap of the persistence of the agonist-AT1R contacts for all the ten peptide agonists highlights the presence of AT1R residue contacts that are selectively sampled only by $\beta$-arr2 biased agonists and others only by $\mathrm{Gq}$ biased agonists. The $\beta$-arr2 biased ligand contacts with AT1R that confer functional selectivity are located mainly on TM7. We calculated the distance distributions of the three well characterized activation microswitches, namely the PIF motif on TM5-TM6 interface, sodium binding site and inwards movement of Y302 ${ }^{7.53}$. As anticipated AT1R is more flexibile in the MD simulations when bound to an agonist compared to when bound to an antagonist. The three activation microswitches show different levels of activation for the ten agonists as can be anticipated when calculated using an ensemble of conformations, suggesting a rheostat like behavior rather than binary on and off switches.

\section{Materials and Methods}

Receptor and agonist preparation for AT1R-agonist system setup: All MD simulations of wild type AT1R in the active and inactive states bound to the unbiased, $\beta$-arr2 biased and $\mathrm{Gq}$ protein biased agonists (Table S1) were performed using the GROMACS package (37) with the CHARMM36 forcefield (38) for proteins, POPC lipids, ions, and using CHARMM TIP3P water as solvent. MD simulations of ten agonists bound to the fully active state of AT1R were performed for Angll, SII, TRV023, TRV026, TRV027, TRV034, TRV044, TRV045, TRV055, and TRV056. The starting structure was taken from the active state structure of S1I8 bound AT1R (PDB: 6DO1) (12). Starting agonist positions for all ten AT1R-agonist complexes were obtained by mutating the corresponding residues back from the native ligand S1I8 in PDB: 6DO1 (see Supplementary Information). The amino acid sequence of all the Angll derivative peptides used in this study and their experimental bias factors are shown in Table S1. The protein structure was prepared and equilibrated for 50ns using procedure described in the Supplementary Information. The equilibration step was followed by 5 production runs with different starting velocities each 400ns long. The snapshots were stored every 20ps and the entire 400ns x 5 runs amounting to $2 \mu$ s of simulation time per system was used for analysis. Details of the MD simulations and the systems are listed in Table S2. Tests for convergence of the MD simulations were done using overlap between principal components (Fig. S9), methods used to cluster the conformations and other MD analyses are provided in Supplementary information text.

Method to calculate Allosteric Communication and Ligand Bias: We used the Allosteer method $(16,17,39)$ to identify the network of residues in the allosteric communication to $\mathrm{Gq}$ and $\beta$-arr2 coupling sites. Using the aggregated trajectories from MD simulations adding to $1 \mu \mathrm{s}$ for each agonist bound AT1R, we calculated the mutual information in torsion angle distributions for all pairs of residues in the receptor (see Fig. S10 for a flow chart of this procedure). For the residue pairs in the top $10 \%$ of the mutual information, we calculated the shortest allosteric communication pathway from the extracellular region of the receptor traversing through the residues in the agonist 
binding site to the $\mathrm{Gq}$ protein or $\beta$-arr2 coupling interface residues. See text in Supporting Information for more details. For each residue in AT1R, the number of allosteric communication pathways passing through them is called the "hubscore". This quantifies the contribution of that residue to the allosteric communication to the $\beta$-arr2 and Gq protein interfaces. The residues in the $\beta$-arr2 and $\mathrm{Gq}$ protein coupling interfaces were taken by translating this information from other solved $\mathrm{Gq}$ protein and $\beta$-arr2 bound GPCR complex structures. The list of AT1R residues posited to be in the $\beta$-arr2 and Gq protein coupling interface used in this study are listed in Table S4. For each residue we calculated the difference in hub score to $\mathrm{Gq}$ coupling site and $\beta$-arr2 coupling site. We performed quartile analysis of this difference in hub score for identifying Gq protein hubs. As seen in Fig. S2, those residues in the last quartile of this hub score distribution were predicted to have either increased or decreased receptor activity to $\mathrm{Gq}$ protein coupling upon mutation. For the prediction of $\beta$-arr2 hubs, we used the hub score distribution of all residues to the $\beta$-arr2 coupling site and employed the last two quartiles to identify $\beta$-arr2 hubs in the Angll-AT1R system. We also used Allosteer to calculate the ligand bias as described in the Supplementary Information.

Inter-residue distance measurements for activation microswitches and generation of heatmap for activation microswitches: The three inter-residue distances $\mathrm{D} 74^{2.50}-\mathrm{N} 111^{3.35}$, D74 ${ }^{2.50}-\mathrm{N} 295^{7.46}$ and $\mathrm{N} 111^{3.35}$ $\mathrm{N} 295^{7.46}$ for defining the sodium binding site were calculated as the minimum distance between the side chain heavy atoms of the participating residues, i.e., CB, CG, OD1, OD2 for aspartate, and CB, CG, OD1, ND2 for asparagine. The distance between the $\mathrm{Y} 215^{5.58}$ and $\mathrm{Y} 302^{7.53}$ is a microswitch measured between the hydroxyl oxygen atoms, i.e., $\mathrm{OH}$, of the two tyrosine residues. The PF microswitch is measured as the minimum distance between any of the carbon atoms in the side chains of P2075.50 i.e., CB, CD, CG and of F2496.44 i.e. CB, CG, CD1, CD2, CE1, CE2. These measurements were carried out using the mindist module in GROMACS (37). More details are given in the Supporting Information.

Ligand Receptor contact frequency heatmap generation: The heatmap shown in Fig. S7B were generated for all receptor-ligand contacts using GetContacts (40). The pie charts (Fig. 3A) were constructed by calculating the difference in average persistence frequencies of each contact across all ßarr2-biased agonists and all Gq-biased agonists. If the calculated difference in the average frequency of a contact is $\geq 10 \%$ for $\beta$-arr2 compared to Gq agonists, it has been counted as having a higher persistence frequency with either ßarr2-biased agonists or Gqbiased agonists. For Figures 3B and 3C, the contacts in TRV026 or TRV056 that had a higher persistence frequency compared to Angll of $\geq 10 \%$ have been shown, with those residues in brown indicating contacts uniquely formed in TRV026 or TRV056.

Site-directed receptor mutagenesis: A two-fragment PCR approach was used for the mutagenesis, as described previously (41). Briefly, site-directed mutagenesis primers were generated with 18bp of Gibson homology for Gibson assembly recombination to insert alanine mutations into the signal peptide-FLAG-tagged-AT1R (42). Mutations were made through stepdown PCR, where two separate PCRs were run to split the vector in half. Each half of PCR samples were then purified, and Gibson ligated. The re-annealed vector was transformed into $E$. coli and one colony was picked and amplified. Plasmid DNA was purified using QIAprep Spin Miniprep kit (QIAGEN) and sequenced to verify successful mutagenesis.

BRET Assay: To measure Gaq or $\beta$-arr2 activation, HEK293SL cells were co-transfected with WT or mutant receptors along with either PKC-c1b BRET sensor (19), or $\beta$-arr2-Rlucll and the plasma membraned-anchored rGFP-CAAX (22), respectively. Cells were transfected and seeded on poly-ornithine-coated, 96-well white plates (BrandTech). 48 hours later, cells were incubated with Tyrodes buffer (140 mM NaCl, $2.7 \mathrm{mM} \mathrm{KCl}, 1 \mathrm{mM} \mathrm{CaCl} 2,12$ $\mathrm{mM} \mathrm{NaHCO}_{3}, 5.6 \mathrm{mM}$ D-glucose, $0.5 \mathrm{mM} \mathrm{MgCl}_{2}, 0.37 \mathrm{mM} \mathrm{NaH}_{2} \mathrm{PO}_{4}, 25 \mathrm{mM} \mathrm{HEPES}, \mathrm{pH} 7.4$ ) at room temperature for 1 hour. Cells were subsequently stimulated with various concentrations of Angll $\left(10^{-12} M-12^{-5} M\right)$ for 2 min to induce maximal receptor activation and generate concentration-response curves to calculate EC50 and Emax for Gaq or $\beta$-arr2 activation. The Rluc substrate coelenterazine 400a (2.5 $\mu \mathrm{M}$; NanoLight Technology) was added 3 min before BRET measurement. BRET measurements were performed using the Synergy 2 microplate reader (BioTek) with donor filter $(410 \pm 80 \mathrm{~nm})$ and acceptor filter $(515 \pm 30 \mathrm{~nm})$. BRET ratios were calculated by dividing the intensity of signal emitted by acceptor over the signal emitted by donor, and BRET change was calculated as the difference between Angll-promoted BRET ratio and unstimulated BRET ratio for each receptor.

Whole-cell ELISA: To measure cell surface expression of receptors, HEK293SL cells were transfected with WT or mutant receptors and seeded on poly-L-lysine-coated, 96-well clear plates (Fisher Scientific). 48 hours later, cells were washed with PBS and fixed with $4 \%$ PFA. Cells were then blocked with $2 \%$ BSA and incubated with antiFLAG M2-peroxidase (Sigma). Cells were washed and colorimetric HRP substrate (SIGMAFAST OPD) was added into each well. After 10 min incubation, $3 \mathrm{M} \mathrm{HCL}$ was added to stop the reaction. The plate was then read at an absorbance of $492 \mathrm{~nm}$ using Synergy 2 microplate reader (BioTek). To obtain specific signal, non-specific signal from pcDNA-transfected cells was subtracted.

\section{Acknowledgements}


bioRxiv preprint doi: https://doi.org/10.1101/2021.05.17.444454; this version posted May 17, 2021. The copyright holder for this preprint (which was not certified by peer review) is the author/funder. All rights reserved. No reuse allowed without permission.

This work was funded by grants from the National Institute of Health (2R01-GM097261) to N.V. and the Canadian Institutes of Health Research (CIHR, MOP-74603) to S.A.L. Y.C. is supported by a doctoral training scholarship from the Fonds de Recherche Santé Quebéc. 


\section{References}

1. S. K. Shenoy, R. J. Lefkowitz, Angiotensin II-stimulated signaling through G proteins and $\beta$ arrestin. Science Signaling 2005, cm14-cm14 (2005).

2. L. B. Teixeira, et al., Ang-(1-7) is an endogenous $\beta$-arrestin-biased agonist of the AT 1 receptor with protective action in cardiac hypertrophy. Scientific Reports 7, 1-10 (2017).

3. J. W. Wisler, K. Xiao, A. R. Thomsen, R. J. Lefkowitz, Recent developments in biased agonism. Current opinion in cell biology 27, 18-24 (2014).

4. S. Rajagopal, K. Rajagopal, R. J. Lefkowitz, Teaching old receptors new tricks: biasing seventransmembrane receptors. Nature reviews Drug discovery 9, 373-386 (2010).

5. J. D. Urban, et al., Functional selectivity and classical concepts of quantitative pharmacology. Journal of Pharmacology and Experimental Therapeutics 320, 1-13 (2007).

6. A. K. Shukla, G. Singh, E. Ghosh, Emerging structural insights into biased GPCR signaling. Trends in biochemical sciences 39, 594-602 (2014).

7. L. M. Luttrell, S. Maudsley, L. M. Bohn, Fulfilling the promise of" biased" G protein-coupled receptor agonism. Molecular pharmacology 88, 579-588 (2015).

8. M.-H. Lee, et al., The conformational signature of $\beta$-arrestin2 predicts its trafficking and signalling functions. Nature 531, 665-668 (2016).

9. L. Zhou, L. M. Bohn, Functional selectivity of GPCR signaling in animals. Current opinion in cell biology 27, 102-108 (2014).

10. L. M. Wingler, et al., Angiotensin analogs with divergent bias stabilize distinct receptor conformations. Cell 176, 468-478 (2019).

11. W. Li, et al., Single-molecule force spectroscopy study of interactions between angiotensin II type 1 receptor and different biased ligands in living cells. Analytical and bioanalytical chemistry $\mathbf{4 1 0}$, 3275-3284 (2018).

12. L. M. Wingler, C. McMahon, D. P. Staus, R. J. Lefkowitz, A. C. Kruse, Distinctive activation mechanism for angiotensin receptor revealed by a synthetic nanobody. Cell 176, 479-490 (2019).

13. C.-M. Suomivuori, et al., Molecular mechanism of biased signaling in a prototypical G proteincoupled receptor. Science 367, 881-887 (2020).

14. L. M. Wingler, et al., Angiotensin and biased analogs induce structurally distinct active conformations within a GPCR. Science 367, 888-892 (2020).

15. S. Bhattacharya, N. Vaidehi, Differences in allosteric communication pipelines in the inactive and active states of a GPCR. Biophysical journal 107, 422-434 (2014).

16. S. Bhattacharya, R. Salomon-Ferrer, S. Lee, N. Vaidehi, Conserved mechanism of conformational stability and dynamics in G-protein-coupled receptors. Journal of chemical theory and computation 12, 5575-5584 (2016). 
17. A. K. Nivedha, et al., Identifying functional hotspot residues for biased ligand design in G-proteincoupled receptors. Molecular pharmacology 93, 288-296 (2018).

18. S. Lee, A. K. Nivedha, C. G. Tate, N. Vaidehi, Dynamic role of the G protein in stabilizing the active state of the adenosine A2A receptor. Structure 27, 703-712 (2019).

19. Y. Namkung, et al., Functional selectivity profiling of the angiotensin II type 1 receptor using pathway-wide BRET signaling sensors. Science signaling 11 (2018).

20. B. Zimmerman, et al., Differential $\beta$-Arrestin-Dependent Conformational Signaling and Cellular Responses Revealed by Angiotensin Analogs. Science signaling 5, ra33-ra33 (2012).

21. Y. Namkung, et al., Quantifying biased signaling in GPCRs using BRET-based biosensors. Methods 92, 5-10 (2016).

22. Y. Namkung, et al., Monitoring G protein-coupled receptor and $\beta$-arrestin trafficking in live cells using enhanced bystander BRET. Nature communications 7, 1-12 (2016).

23. A. Beautrait, et al., A new inhibitor of the $\beta$-arrestin/AP2 endocytic complex reveals interplay between GPCR internalization and signalling. Nature communications 8, 1-16 (2017).

24. C. S. Tautermann, et al., Allosteric activation of striatal-enriched protein tyrosine phosphatase (STEP, PTPN5) by a fragment-like molecule. Journal of medicinal chemistry 62, 306-316 (2018).

25. R. Bhargava, et al., C-NHEJ without indels is robust and requires synergistic function of distinct XLF domains. Nature communications 9, 1-14 (2018).

26. S. Maeda, Q. Qu, M. J. Robertson, G. Skiniotis, B. K. Kobilka, Structures of the M1 and M2 muscarinic acetylcholine receptor/G-protein complexes. Science 364, 552-557 (2019).

27. L. Yang, et al., Molecular Basis of $\beta$-arrestin Coupling to Formoterol-Bound $\beta$ 1-adrenoceptor. Nature.

28. J. C. Gomez Tamayo, et al., GPCR-SAS: A web application for statistical analyses on G proteincoupled receptors sequences. Plos one 13, e0199843 (2018).

29. R. T. Strachan, et al., Divergent transducer-specific molecular efficacies generate biased agonism at a $\mathrm{G}$ protein-coupled receptor (GPCR). Journal of Biological Chemistry 289, 14211-14224 (2014).

30. S. Rajagopal, et al., Quantifying ligand bias at seven-transmembrane receptors. Molecular pharmacology 80, 367-377 (2011).

31. K. L. White, et al., Structural connection between activation microswitch and allosteric sodium site in GPCR signaling. Structure 26, 259-269 (2018).

32. B. G. Tehan, A. Bortolato, F. E. Blaney, M. P. Weir, J. S. Mason, Unifying family A GPCR theories of activation. Pharmacology \& therapeutics 143, 51-60 (2014).

33. W. Liu, et al., Structural basis for allosteric regulation of GPCRs by sodium ions. Science 337, 232-236 (2012). 
34. N. Ma, S. Lee, N. Vaidehi, Activation Microswitches in Adenosine Receptor A2A Function as Rheostats in the Cell Membrane. Biochemistry (2020).

35. C. Lee, et al., Manifold active-state conformations in GPCRs: agonist-activated constitutively active mutant AT1 receptor preferentially couples to Gq compared to the wild-type AT1 receptor. FEBS letters 581, 2517-2522 (2007).

36. M. M. Bonde, et al., Biased signaling of the angiotensin II type 1 receptor can be mediated through distinct mechanisms. PLoS One 5, e14135 (2010).

37. H. J. Berendsen, D. van der Spoel, R. van Drunen, GROMACS: a message-passing parallel molecular dynamics implementation. Computer physics communications 91, 43-56 (1995).

38. J. Huang, A. D. MacKerell Jr, CHARMM36 all-atom additive protein force field: Validation based on comparison to NMR data. Journal of computational chemistry 34, 2135-2145 (2013).

39. N. Vaidehi, S. Bhattacharya, Allosteric communication pipelines in G-protein-coupled receptors. Current opinion in pharmacology 30, 76-83 (2016).

40., GetContacts: Library for computing dynamic non-covalent contact networks in proteins throughout MD Simulation.

41. F. M. Heydenreich, et al., High-throughput mutagenesis using a two-fragment PCR approach. Scientific reports 7, 1-11 (2017).

42. E. Goupil, et al., Angiotensin II type I and prostaglandin F2 $\alpha$ receptors cooperatively modulate signaling in vascular smooth muscle cells. Journal of Biological Chemistry 290, 3137-3148 (2015). 